\title{
Asociación entre espacio de vida y factores psicológicos y funcionales en personas mayores de Brasil y Colombia
}

\section{Association between living space and psychological and functional factors in elderly people in Brazil and Colombia}

DOI: $10.46932 / \mathrm{sfjdv} 3 \mathrm{n} 1-020$

Received in: Dec 30st, 2021

Accepted in: Jan 1th, 2022

\section{Carmen-Lucia Curcio}

Terapeuta Ocupacional.

Ph.D.

Gerontología.

Grupo de Investigaciones en Gerontología y Geriatría.

Facultad de Ciencias para la Salud, Universidad de Caldas.

E-mail: carmen.curcio@ucaldas.edu.co

\section{Manuel Pérez-Trujillo}

Ingeniero electrónico.

Mgr en Administración de Sistemas Informáticos.

Grupo de investigación en Ambientes Inteligentes Adaptativos GAIA. Departamento de Informática y Computación.

Universidad Nacional de Colombia. Manizales.

\section{Ricardo Guerra}

Fisioterapeuta.

Ph.D.

epidemiologia del envejecimiento.

Departamento de Fisioterapia, Universidad Federal del Rio Grande del Norte. Natal. Brazil

\section{Cristiano Gomes}

Fisioterapeuta.

Ph.D.

Fisioterapia.

Departamento de Fisioterapia, Universidad Federal del Rio Grande del Norte. Natal. Brazil

\section{Néstor Duque-Méndez \\ Ingeniero mecánico.}

Ph.D.

Ingeniería.

Grupo de investigación en Ambientes Inteligentes Adaptativos GAIA.

Departamento de Informática y Computación.

Universidad Nacional de Colombia. Manizales 


\title{
RESUMEN
}

Objetivo: examinar la asociación entre la movilidad en el espacio de vida y las diferentes dimensiones de los síntomas depresivos y la funcionalidad a partir de datos obtenidos en estudio de ancianos que viven en la comunidad de Brasil y Colombia. Materiales y Métodos: estudio transversal y correlacional en el cual se realizó un análisis secundario a partir del estudio IMIAS, se tomó información de Manizales (Colombia) y Natal (Brasil) del 2014. La muestra fue de 666 personas mayores. Se utilizó el cuestionario de evaluación de la movilidad en el espacio de vida; para la depresión y sus dimensiones se usó la escala CES-D. La capacidad funcional se midió con la prueba SPPB. Se hizo un análisis por ciudad y sexo, en donde la comparación entre sexos se llevó a cabo usando las pruebas de Mann-Whitney y chi-cuadrada. Para predecir espacio de vida se hicieron 3 modelos de regresión lineal y 3 para un análisis no lineal mediante árboles de decisión. Resultados: Los promedios de espacio de vida son bajos, en Manizales fue 68.51 y en Natal 52.88. En los dos sitios las diferentes dimensiones de depresión y edad son predictores importantes en ambos sexos. En Manizales la edad, el afecto deprimido, síntomas somáticos y afecto positivo, predicen la restricción del espacio de vida y, puntualmente para mujeres, se agrega el SPPB. Para Natal se evidenció una asociación en ambos sexos según los síntomas somáticos, afecto positivo, la capacidad funcional y la discapacidad. Conclusión: En los dos sitios la depresión, funcionalidad, edad y la discapacidad de movilidad son importantes predictores de restricción del espacio de vida principalmente en mujeres. El espacio de vida tiene predictores diferenciales según el sitio y el sexo.

Palabras clave: depresión, espacio de vida, movilidad, ancianos, árboles de decision.

\begin{abstract}
Aim: to examine the association between life-space mobility and the different dimensions of depressive symptoms and functionality among older people living in the community of Brazil and Colombia. Methods: A cross-sectional and a correlational study in which a secondary analysis was carried out for the IMIAS study, based on information from Manizales (Colombia) and Natal (Brazil) of 2014. A scale with 20 items and total score of 0 to 120 was used to assess life space while depression and its dimensions were measured by the CES-D scale. Functional capacity was measured with SPPB test. A description of the population was drawn up for both cities grouped by sex. The comparison between the sexes for each variable was carried out with Mann-Whitney and chi-square tests for continuous and categorical variables, respectively. Finally, 3 linear regression models and decision trees were used to predict life-space. Results: Mean scores of life-space is low, in Manizales it was 68.51 and in Natal 52.88. In both sites, dimensions of depression and age are important predictors of life space mobility in both sexes. In Manizales, age, depressive affect, somatic complaint and, positive affect, predicts the restriction of lifespace and, SPPB is added for women. Was evident the association in both sexes according to somatic complaint, positive affect, functional capacity and mobility disability. Conclusion: In both sites, depression, functional capacity, age and mobility disability are important predictors of poorer life space mobility, mainly in women. The living space has differential predictors according to site and sex.
\end{abstract}

Keywords: depression, Life-space, mobility, elderly, decision trees.

\section{INTRODUCCIÓN}

La movilidad conservada es fundamental para un envejecimiento saludable, ya que permite a las personas mayores llevar una vida activa autónoma e independiente. Una forma útil de ver el funcionamiento y la participación de las personas mayores en el mundo real es a través de la movilidad 
en el espacio de vida, es decir, el movimiento dentro y entre diversos ambientes (Allman et al., 2006; Giannouli et al., 2019; Peel et al., 2005). La movilidad en el espacio de vida refleja la ejecución real y se refiere al área espacial en la que una persona se mueve en la vida cotidiana, la frecuencia con que lo hace y la necesidad de asistencia (Allman et al., 2006; Baker et al., 2003; Stalvey et al., 1999). La movilidad en el espacio de vida no solo mide la capacidad de caminar de las personas, sino que también incluye otras formas de movilidad, como usar el transporte público o conducir un automóvil (Béland et al., 2018; Stalvey et al., 1999) y es un indicador de participación social (Sawyer \& Allman, 2010). Un espacio de vida (EV) más amplio proporciona a un individuo más oportunidades de relacionarse con la sociedad (Kono et al., 2004; Kuspinar et al., 2020) mientras que la restricción de este espacio de vida puede reflejar un acceso limitado a los servicios sociales y se correlaciona positivamente con la calidad de vida (Rantakokko et al., 2015) y participación social activa (Barnes et al., 2007; Taylor et al., 2018). La restricción del espacio de vida se asocia con una pobre ejecución física, limitaciones de movilidad y discapacidad (Baker et al., 2003; C. L. Curcio et al., 2013; Portegijs et al., 2015, 2016), reducido sentido de la autonomía(Portegijs et al., 2014), síntomas depresivos (Polku et al., 2015), deterioro cognoscitivo (James et al., 2011) y fragilidad (Xue et al., 2008). También, se ha reportado menor espacio de vida en las mujeres (C. L. Curcio et al., 2013; Sawyer \& Allman, 2010).

La Organización Mundial de la Salud (OMS) reporta la depresión como el trastorno mental de mayor ocurrencia en los ancianos (Organización Mundial de la Salud (OMS), 2017). Aproximadamente un $20 \%$ de los adultos de 60 años o más sufren algún trastorno mental o neurológico y la depresión afecta a un 7\% de la población anciana en general (Organización Mundial de la Salud (OMS), 2017). En casi todo el mundo, la depresión es dos o tres veces más común en las mujeres (Eugenia Alvarado et al., 2007; Nolen-Hoeksema, 2001; Vaughan et al., 2015; M. V. Zunzunegui et al., 1998). Sin embargo, la prevalencia de depresión varía entre países. Un estudio transversal internacional llevado a cabo en 4 países muestra tanto las diferencias por sitio como por sexo. En Tirana la prevalencia fue $27,1 \%$ en hombres y 46,6\% en mujeres, seguida de Manizales (17,7\% en hombres y 28,7\% en mujeres) y Natal (6,3\% en hombres y $31,9 \%$ en mujeres); los dos sitios canadienses incluidos en el estudio tuvieron las tasas de prevalencia más bajas, 10,5\% en Saint-Hyacinthe y 9,1\% en Kingston (Ylli et al., 2016).

El concepto de EV reconoce que la movilidad puede verse afectada por múltiples factores y no solamente por la función física (Allman et al., 2006; Bentley et al., 2013). Se ha establecido que, una movilidad más restringida en el espacio de vida se correlaciona con una mayor probabilidad de síntomas depresivos (Baker et al., 2003; Béland et al., 2018; Boyle et al., 2010; C. L. Curcio et al., 2013; Peel et al., 2005; Polku et al., 2015; Snih et al., 2012). Estar confinado en casa es un predictor significativo de depresión en ancianos (Cohen-Mansfield et al., 2010). Con respecto a los dominios de la depresión, solo 
se encontró un estudio que ha analizado los cuatro factores medidos en la escala CES-D y su relación con espacio de vida y muestra que las asociaciones entre las diferentes dimensiones de los síntomas depresivos y la movilidad en el espacio de vida no son directas, sino que están mediadas en parte por dificultades para caminar, condiciones crónicas y autonomía para la participación en actividades al aire libre (Polku et al., 2015).

De otro lado, las personas con un EV más restringido o aquellas que dependen de dispositivos de asistencia o asistencia personal para actividades fuera del hogar pueden tener menos oportunidades de participar en actividades significativas en la comunidad (Baker et al., 2003). Sin embargo, no necesariamente perciben sus posibilidades como restringidas (Barnes et al., 2007; Cardol et al., 1999), pero si pueden compensar los déficits utilizando los recursos disponibles (por ejemplo, transporte, apoyo social) (Viljanen et al., 2016) o si valoran objetivos personales como relevantes (Saajanaho et al., 2015).

En un estudio llevado a cabo en Manizales y Natal se encontró que el espacio de vida se relaciona con ejecución física, limitaciones en movilidad y discapacidad (C. L. Curcio et al., 2013). En otro estudio, el puntaje de movilidad en el espacio de vida fue el más alto y se mantuvo estable durante 2 años entre las personas que no tenían dificultades para caminar al inicio del estudio, y los puntajes fueron más bajos y con una disminución constante entre aquellos que si las tenían (Rantakokko et al., 2017), así mismo, cambios en la marcha se asocian con disminución en $\operatorname{EV}(9,35)$.

Dada la importancia de la movilidad para el funcionamiento independiente y los efectos adversos de su restricción, el objetivo de este estudio fue examinar la asociación entre la movilidad en el espacio de vida y las diferentes dimensiones de los síntomas depresivos y la funcionalidad entre personas mayores que viven en la comunidad de Brasil y Colombia.

\section{MATERIALES Y MÉTODOS}

Tipo de estudio y diseño: estudio transversal y correlacional anidado en el International Mobility in Aging Study (IMIAS), una investigación que estudia los cambios de la movilidad al envejecer (Gomez et al., 2018).

Población y muestra: Se tomó información de Manizales (Colombia) y Natal (Brasil) del 2014 y se incluyeron solamente aquellos registros con los datos completos, 353 personas en Manizales y 313 de Natal.

Variable dependiente: Espacio de vida - EV: se utilizó el cuestionario de evaluación del espacio de vida (Peel et al., 2005), que se refiere al área espacial en la que una persona se mueve en la vida cotidiana, la frecuencia con que lo hace y la necesidad de asistencia. Tiene un total de 20 ítems y el puntaje total va de 0 a 120 . Su validez y confiabilidad en español y portugués fue previamente establecida (C. L. 
Curcio et al., 2013). Altos puntajes indican amplios espacios de vida, puntajes inferiores a 60 indican restricción del espacio de vida $(7,37)$.

\section{COVARIABLES}

Los aspectos demográficos incluyeron edad, sexo, nivel de escolaridad, nivel de ingresos y vivir solo. Los aspectos psicológicos incluyen las diferentes dimensiones de depresión. La depresión se midió con la escala CES-D (Ruiz-Grosso et al., 2012) validada para población colombiana (Campo-Arias et al., 2007). Es una escala de tamización de sintomatología depresiva, compuesta por veinte ítems que dan cuenta de 4 dimensiones: afecto deprimido - AD (7 ítems), síntomas somáticos - SS (7 ítems), afecto positivo - AP (4 ítems) y relaciones interpersonales - RI (2 ítems), y reflejan el estado de ánimo del sujeto durante la semana previa. Se midió la frecuencia de los síntomas en cada dimensión (1= nunca o rara vez; $2=$ algunas veces; $3=$ con frecuencia; $4=$ siempre). Los puntajes totales están entre 0 y 60 y se utilizó el punto de corte de 16 sugestivo de depresión (Thomas et al., 2001).

El aspecto funcional involucró la prueba Short Physical Performance Battery (SPPB), que consta de 3 subpruebas: equilibrio, velocidad de la marcha e incorporarse de una silla (Guralnik et al., 1994). En cada prueba se puede obtener un máximo de 4. La calificación total es la sumatoria y está comprendida entre 0 y 12, siendo 12 el mejor rendimiento. Para la discapacidad de movilidad se utilizó el auto reporte de dificultad en caminar $400 \mathrm{~m}$ (Nagi, 1976).

\section{ANÁLISIS ESTADÍSTICO}

Se hizo una descripción de la población para ambas ciudades agrupadas por sexo. Las características en función de las variables continuas se describieron usando la media, mediana y los rangos intercuartílicos (IQR). Para las categóricas, la descripción se realizó usando el porcentaje y la frecuencia. La comparación entre sexos de los análisis para cada variable se realizó con las pruebas Mann-Whitney y $\chi^{2}$ para las variables continuas y categóricas, respectivamente. Para las correlaciones se utilizaron las pruebas de Pearson y Spearman según la naturaleza de las variables.

Se hizo un ajuste de regresiones lineales para predecir el EV. A través de la prueba de Wald se halló la importancia que tenía cada una de las dimensiones de CES-D o SPPB, según cada modelo. En total se presentaron 3 modelos para predecir el espacio de vida. En el primero se incluyeron las variables edad, situación financiera y depresión o SPPB. El segundo modelo incluyó las variables del primer modelo junto con discapacidad de movilidad. El tercero incluyó las variables del modelo 2 junto con la variable que informaba si la persona vivía sola. Éstos 3 modelos se evaluaron para hombres y mujeres de manera 
independiente. La depresión se analizó en 5 dimensiones: puntaje total, afecto deprimido, síntomas somáticos, afecto positivo y relaciones interpersonales.

Se llevó a cabo un análisis no lineal mediante árboles de decisión (Villarreal et al., 2011). Se utilizaron los mismos modelos y metodología mencionados arriba para las regresiones lineales. El análisis consistió en la predicción del espacio de vida restringido. El entrenamiento del algoritmo se realizó con el $80 \%$ de los datos de cada ciudad, se validó con el 20\% restante. El rendimiento del algoritmo de cada uno de los modelos ajustados se valoró con el Área Bajo la Curva (AUC por sus siglas en inglés) de la Característica Operativa del Receptor (ROC por sus siglas en inglés) (Huang \& Ling, 2005). Aquellos ajustes con un AUC-ROC aceptable (entre 0,7 y 0,8$)$ y excelente $(>0,8)$ se analizaron para determinar las variables de mayor importancia en la predicción del EV.

Los análisis estadísticos se realizaron con el software SPSS v. 23 y Anaconda v.1.9.12 usando Python. Para el árbol de decisión se utilizó el algoritmo CART. El proceso de entrenamiento y testeo se hizo a través de los paquetes suministrados por Scikit Learn (Pedregosa et al., 2011).

Consideraciones éticas: el estudio es aprobado por los Comités de Ética de las universidades de Caldas (Colombia) y del Rio Grande del Norte (Brasil). Todos los participantes firmaron un consentimiento informado, a fin de cumplir con los principios éticos de la Asociación Médica Mundial (Declaración de Helsinki) y la normativa vigente.

\section{RESULTADOS}

La muestra total fue de 353 personas mayores en Manizales y 313 en Natal. En ambas ciudades la media de edad en las mujeres fue superior a la de los hombres. Con respecto a los años de educación, Manizales tuvo una población más educada con respecto a Natal, con 6.55 años para los hombres y 5.30 años para las mujeres, así mismo, fue la ciudad con mayor proporción de personas que viven sola (14.16\%) y la de mayor población pobre $(47.87 \%)$.

El promedio de EV en Manizales fue de 68.68 y, aunque es bajo, no se puede considerar restringido y los hombres tienen mayor espacio de vida en comparación con las mujeres (72.91 vs. 64.57, p<.001). En Natal el promedio de EV fue menor con 53.36 y tanto hombres como mujeres tienen un EV restringido, esto es, inferior a 60. En cuanto a la depresión, las mujeres tienen un promedio mayor en depresión en ambas ciudades. De igual forma, las mujeres tienen una media más alta en comparación con los hombres de los dos sitios en las cuatro dimensiones de depresión, tal como se muestra en la Tabla 1. 
Tabla 1. Características de los participantes en Manizales y Natal según sexo.

\begin{tabular}{|c|c|c|c|c|c|c|c|c|c|c|c|c|c|c|}
\hline & \multicolumn{7}{|c|}{ Manizales (353) } & \multicolumn{7}{|c|}{ Natal (313) } \\
\hline & \multicolumn{3}{|c|}{ Hombres (174) } & \multicolumn{3}{|c|}{ Mujeres (179) } & $p^{a}$ & \multicolumn{3}{|c|}{ Hombres (148) } & \multicolumn{3}{|c|}{ Mujeres (165) } & $p^{a}$ \\
\hline Edad & 71.39 & 71 & 5 & 71.51 & 72 & 5 & 0.67 & 71.48 & 72 & 5 & 71.62 & 72 & 5 & 0.67 \\
\hline $\begin{array}{l}\text { Espacio de } \\
\text { vida }\end{array}$ & 72.91 & 74 & 17.5 & 64.57 & 64 & 18 & $<.001$ & 59.50 & 58 & 24 & 47.87 & 48 & 18 & $<.001$ \\
\hline Depresión & 6 & 4 & 7 & 9.03 & 6 & 13 & $<.001$ & 7.10 & 5.5 & 9 & 10.88 & 9 & 9 & $<.001$ \\
\hline $\begin{array}{l}\text { Síntomas } \\
\text { somáticos }\end{array}$ & 2.04 & 1 & 3 & 3.30 & 2 & 4 & $<.001$ & 2.37 & 2 & 4 & 3.68 & 3 & 4 & $<.001$ \\
\hline $\begin{array}{l}\text { Afecto } \\
\text { positivo }\end{array}$ & 1.87 & 1 & 3 & 2.46 & 2 & 4 & 0.02 & 2.56 & 1 & 5 & 2.92 & 2 & 5 & 0.12 \\
\hline $\begin{array}{l}\text { Relaciones } \\
\text { interpersonales }\end{array}$ & 0.32 & 0 & 0 & 0.44 & 0 & 0.5 & 0.10 & 0.45 & 0 & 1 & 0.53 & 0 & 1 & 0.81 \\
\hline $\begin{array}{l}\text { Situación } \\
\text { financiera }\end{array}$ & & & & & & & $<.001$ & & & & & & & $<.001$ \\
\hline $\begin{array}{l}\text { Buena o } \\
\text { muy Buena }\end{array}$ & 32.18 & 56 & & 11.17 & 20 & & & 56.0 & 83 & & 30.3 & 50 & & \\
\hline Moderada & 32.18 & 56 & & 29.05 & 52 & & & 40.5 & 60 & & 57.5 & 95 & & \\
\hline Pobre & 35.63 & 62 & & 59.77 & 107 & & & 3.37 & 5 & & 12.1 & 20 & & \\
\hline
\end{tabular}

SPPB $=$ Short Physical Performance Battery. IQR $=$ Rangos intercuartílicos. $p^{a}=$ significancia prueba Mann-Whitney. $p^{b}=$ significancia prueba $\chi^{2}$.

En Manizales, el EV se correlacionó significativamente con la depresión $(\mathrm{p}<0.01)$ tanto en hombres como mujeres (h:-0.266, m:-0.259), con discapacidad de movilidad (h:-0.226, m:-0.302), con SPPB (h:0.327, m:0.408), con SS (h:-0.201, m:-0.288) y con AP (h:-0.334, m:-0.246). Asimismo, en los hombres la correlación de EV con discapacidad de movilidad (-0.226) y con los ingresos económicos (0.384) fue significativa ( $\mathrm{p}<0.01)$. Por su parte, en Natal las correlaciones fueron significativas en ambos sexos entre el EV con SPPB (h:0.382, m:0.464) y con discapacidad de movilidad (h:-0.247, m:-0.396).

Las asociaciones lineales entre las diferentes dimensiones de la depresión y la funcionalidad y el EV se muestran en la Tabla 2 para ambas ciudades. Para Manizales, en el primer modelo se identificó una relación significativa para todas las dimensiones de depresión y el SPPB en las mujeres, exceptuando la dimensión de relaciones interpersonales. Específicamente, una alta significancia en el puntaje total de depresión, síntomas somáticos y SPPB $(\mathrm{p}<0.001)$. En los hombres las dimensiones que tuvieron significancia fueron el puntaje total de depresión, afecto positivo y SPPB, siendo estas dos últimas las de más significancia $(\mathrm{p}<0.01)$. Para el segundo modelo (controlado por discapacidad) y el tercer modelo (controlado con discapacidad y si vive solo), las variables que mantuvieron su significancia fueron síntomas somáticos y SPPB en el sexo femenino. 
En el caso de Natal, las asociaciones lineales muestran que en el primer modelo solamente fueron significativos los síntomas somáticos y el SPPB en ambos sexos. Específicamente para las mujeres se identificó una significancia adicional con respecto a la calificación total de depresión y la dimensión afecto deprimido. No se observó significancia en las diferentes dimensiones de depresión para ninguno de los sexos en los demás modelos, exceptuando el SPPB con significancia alta $(\mathrm{p}<0.001)$.

En la Tabla 3 se muestra las asociaciones no lineales. En Manizales se obtuvieron 12 ajustes con un AUC-ROC aceptable (0.7 - 0.8) (Mandrekar, 2010). De los doce ajustes, las variables con mayor poder predictivo de restricción de EV son discapacidad y edad (Tabla 3). De las dimensiones de la depresión, el afecto deprimido, el afecto positivo y el SPPB predicen la restricción del EV, logrando en algunos casos una importancia de hasta $45.5 \%$ en sus respectivos modelos.

En Natal se obtuvieron 3 ajustes aceptables (ver Tabla 2), de los cuales 2 fueron en mujeres, en quiénes la restricción del EV se relacionó con la calificación total de la prueba de depresión y la dimensión de afecto positivo, y cada una de estas variables tuvo más de $40 \%$ de importancia en sus respectivos modelos (Tabla 3). En los hombres, el único modelo con AUC-ROC aceptable fue el que incluyó la dimensión de afecto positivo. En los dos sitios, se evidenció que en todos los modelos tanto la discapacidad de movilidad y la edad fueron variables con gran porcentaje de importancia. 
Tabla 2. Asociación lineal y no lineal entre el espacio de vida y las diferentes dimensiones de depresión para hombres y mujeres en Manizales y Natal.

\begin{tabular}{|c|c|c|c|c|c|c|c|c|c|c|c|c|c|c|c|c|c|c|c|}
\hline & \multicolumn{9}{|c|}{ Manizales } & \multicolumn{9}{|c|}{ Natal } \\
\hline & & \multicolumn{3}{|c|}{ Model 1} & \multicolumn{3}{|c|}{ Model 2} & \multicolumn{3}{|c|}{ Model 3} & \multicolumn{3}{|c|}{ Model 1} & \multicolumn{3}{|c|}{ Model 2} & \multicolumn{3}{|c|}{ Model 3} \\
\hline & & $\beta$ & SE & $\begin{array}{c}A U C \\
- \\
R O C\end{array}$ & $\beta$ & SE & $\begin{array}{l}A U C- \\
R O C\end{array}$ & $\beta$ & SE & $\begin{array}{l}A U C- \\
R O C\end{array}$ & $\beta$ & SE & $\begin{array}{c}A U C \\
- \\
R O C\end{array}$ & $\beta$ & SE & $\begin{array}{c}A U C- \\
R O C\end{array}$ & $\beta$ & SE & $\begin{array}{c}A U C- \\
R O C\end{array}$ \\
\hline \multirow[t]{2}{*}{$\begin{array}{l}\text { CES- } \\
\text { D }\end{array}$} & $\mathrm{H}$ & $-0.354 *$ & 0.169 & 0.557 & -0.05 & 0.181 & $\begin{array}{c}0.704 \\
(1)\end{array}$ & -0.082 & 0.185 & 0.681 & -0.214 & 0.213 & 0.459 & 0.011 & 0.215 & 0.517 & -0.009 & 0.220 & 0.524 \\
\hline & M & $0.471 * * *$ & 0.137 & 0.590 & -0.194 & 0.140 & 0.510 & -0.228 & 0.139 & 0.421 & $-0.288^{*}$ & 0.130 & 0.578 & -0.115 & 0.121 & $\begin{array}{c}0.733 \\
(13) \\
\end{array}$ & -0.106 & 0.123 & 0.592 \\
\hline \multirow[t]{2}{*}{$\mathrm{AD}$} & $\mathrm{H}$ & -0.641 & 0.456 & 0.626 & 0.072 & 0.468 & $\begin{array}{c}0.735 \\
(2)\end{array}$ & -0.010 & 0.481 & $\begin{array}{c}0.735 \\
(3)\end{array}$ & 0.007 & 0.494 & 0.500 & 0.410 & 0.485 & 0.468 & 0.377 & 0.503 & 0.453 \\
\hline & M & -0.747* & 0.353 & 0.614 & -0.162 & 0.343 & $\begin{array}{c}0.763 \\
(4)\end{array}$ & -0.239 & 0.340 & $\begin{array}{c}0.744 \\
(5)\end{array}$ & $-0.569 *$ & 0.272 & 0.464 & -0.371 & 0.248 & 0.648 & -0.351 & 0.253 & 0.615 \\
\hline \multirow[t]{2}{*}{ SS } & $\mathrm{H}$ & -0.770 & 0.442 & 0.675 & -0.001 & 0.465 & $\begin{array}{c}\mathbf{0 . 7 5 0} \\
(6)\end{array}$ & -0.079 & 0.476 & $\begin{array}{c}\mathbf{0 . 7 5 0} \\
(7)\end{array}$ & $-1.165^{*}$ & 0.511 & 0.647 & -0.705 & 0.517 & 0.600 & -0.734 & 0.520 & 0.600 \\
\hline & M & - $1.416 * * *$ & 0.364 & 0.649 & $-0.763 *$ & 0.366 & $\begin{array}{c}0.737 \\
(8)\end{array}$ & $-0.768^{*}$ & 0.362 & 0.598 & $-0.775^{*}$ & 0.334 & 0.634 & -0.125 & 0.324 & 0.636 & -0.101 & 0.327 & 0.659 \\
\hline \multirow[t]{2}{*}{$\mathrm{AP}$} & $\mathrm{H}$ & $-1.545 * *$ & 0.525 & 0.652 & -0.798 & 0.551 & $\begin{array}{c}\mathbf{0 . 7 5 8} \\
(9)\end{array}$ & -0.840 & 0.553 & $\begin{array}{c}0.758 \\
(10)\end{array}$ & -0.115 & 0.467 & 0.598 & 0.240 & 0.458 & $\begin{array}{c}\mathbf{0 . 7 2 0} \\
(14)\end{array}$ & 0.221 & 0.462 & 0.649 \\
\hline & M & $-1.505 * *$ & 0.468 & 0.434 & -0.438 & 0.487 & 0.559 & -0.612 & 0.486 & 0.657 & -0.205 & 0.405 & 0.448 & 0.084 & 0.367 & $\begin{array}{c}0.716 \\
(15) \\
\end{array}$ & 0.072 & 0.368 & 0.654 \\
\hline \multirow[t]{2}{*}{ RI } & $\mathrm{H}$ & 0.564 & 1.543 & 0.543 & 1.668 & 1.482 & 0.591 & 1.574 & 1.491 & 0.591 & 0.308 & 1.737 & 0.647 & -0.058 & 1.670 & 0.495 & -0.143 & 1.684 & 0.473 \\
\hline & M & -2.728 & 1.461 & 0.491 & -1.934 & 1.344 & 0.593 & -2.382 & 1.338 & 0.586 & -1.521 & 1.117 & 0.495 & -1.285 & 1.003 & 0.688 & -1.253 & 1.006 & 0.673 \\
\hline \multirow[t]{2}{*}{$\begin{array}{l}\text { SP } \\
\text { PB }\end{array}$} & $\mathrm{H}$ & $2.314 * *$ & 0.739 & 0.669 & 1.203 & 0.789 & $\begin{array}{c}\mathbf{0 . 7 5 5} \\
(11)\end{array}$ & 1.212 & 0.790 & 0.632 & $3.021 * * *$ & 0.628 & 0.645 & $\begin{array}{l}2.512 * * \\
*\end{array}$ & 0.654 & 0.633 & $2.539 * * *$ & 0.656 & 0.633 \\
\hline & M & $4.152 * * *$ & 0.668 & 0.529 & $\begin{array}{l}2.794 * * \\
*\end{array}$ & 0.760 & 0.684 & $2.732 * * *$ & 0.753 & $\begin{array}{c}\mathbf{0 . 7 3 6} \\
(12)\end{array}$ & $2.597 * * *$ & 0.393 & 0.592 & $\begin{array}{l}1.712 * * \\
*\end{array}$ & 0.468 & 0.572 & $1.748 * * *$ & 0.469 & 0.677 \\
\hline
\end{tabular}

CES-D = Calificación total de prueba de depresión. $\mathrm{AD}=$ Afecto deprimido. $\mathrm{SS}=$ Síntomas somáticos. AP = Afecto positivo. $\mathrm{RI}=\mathrm{Rel}$ aciones interpersonales. $\mathrm{B}=$ coeficiente depresivo obtenido en regresión lineal. $\mathrm{SE}=$ error estándar del coeficiente depresivo. AUC-ROC = Área bajo la curva de ROC en ajuste con árbol de decisión. $\mathrm{H}=\mathrm{Hombre}$. $\mathrm{M}$ = Mujer. Negrita = área bajo la curva ROC aceptable o excelente. $(\mathbf{n})=$ número asignado a los mejores ajustes para ser identificados en la Tabla 5 . Modelo 1 = Ajustado por edad, situación financiera y depresión o SPPB. Modelo 2 = ajustado con variables de modelo 1 y dificultades para caminar 400 metros. Modelo $3=$ ajustado con variables de modelo 2 y si vive solo. $* p<0.05, * * p<0.01, * * * p<0.001$ 
Tabla 3. Importancia de las variables predictivas del espacio de vida en los mejores ajustes.

\begin{tabular}{|c|c|c|c|c|c|c|c|}
\hline \multirow{2}{*}{$\begin{array}{l}\text { Número } \\
\text { de ajuste }\end{array}$} & \multicolumn{6}{|c|}{$\%$ de relevancia } & \multirow{2}{*}{$\begin{array}{l}\text { AUC- } \\
\text { ROC }\end{array}$} \\
\hline & Edad & Ingresos & SPPB & $\begin{array}{c}\text { Dimensión } \\
\text { depresión }\end{array}$ & Discapacidad & Vivir solo & \\
\hline \multicolumn{8}{|l|}{ Manizales } \\
\hline $\begin{array}{l}\text { 1. CES-D } \\
\text { hombres }\end{array}$ & 51.3 & 0 & - & 16.7 & 31.9 & - & 0.704 \\
\hline $\begin{array}{c}\text { 2. AD } \\
\text { hombres }\end{array}$ & 32.8 & 0 & - & 34.1 & 32.9 & - & 0.735 \\
\hline $\begin{array}{l}\text { 3. AD } \\
\text { hombres }\end{array}$ & 28.6 & 0 & - & 38.4 & 32.9 & 0 & 0.735 \\
\hline $\begin{array}{l}\text { 4. AD } \\
\text { mujeres }\end{array}$ & 25.8 & 0 & - & 40.7 & 33.3 & - & 0.763 \\
\hline $\begin{array}{l}\text { 5. AD } \\
\text { mujeres }\end{array}$ & 27.5 & 0 & - & 29.4 & 31.9 & 10.9 & 0.744 \\
\hline $\begin{array}{c}\text { 6. SS } \\
\text { hombres }\end{array}$ & 42.6 & 5.4 & - & 22.1 & 29.7 & - & 0.75 \\
\hline $\begin{array}{c}\text { 7. SS } \\
\text { hombres }\end{array}$ & 42.6 & 5.4 & - & 22.1 & 29.7 & 0 & 0.75 \\
\hline $\begin{array}{c}\text { 8. SS } \\
\text { mujeres }\end{array}$ & 25.0 & 15.0 & - & 40.1 & 19.7 & - & 0.737 \\
\hline $\begin{array}{c}\text { 9. AP } \\
\text { hombres }\end{array}$ & 34.8 & 0 & - & 32.7 & 32.3 & - & 0.758 \\
\hline $\begin{array}{c}\text { 10. AP } \\
\text { hombres }\end{array}$ & 34.8 & 0 & - & 32.7 & 32.3 & 0 & 0.758 \\
\hline $\begin{array}{l}\text { 11. SPPB } \\
\text { hombres }\end{array}$ & 48.3 & 0.9 & 12.4 & - & 38.2 & - & 0.755 \\
\hline $\begin{array}{l}\text { 12. SPPB } \\
\text { mujeres }\end{array}$ & 18.7 & 16.3 & 45.5 & - & 11.0 & 8.2 & 0.736 \\
\hline \multicolumn{8}{|l|}{ Natal } \\
\hline $\begin{array}{c}\text { 13. CES- } \\
\text { D } \\
\text { mujeres }\end{array}$ & 4.9 & 25.8 & - & 41.3 & 27.8 & - & 0.733 \\
\hline $\begin{array}{c}\text { 14. AP } \\
\text { hombres }\end{array}$ & 37.4 & 3.8 & - & 39.5 & 19.1 & - & 0.720 \\
\hline $\begin{array}{l}\text { 15. AP } \\
\text { mujeres }\end{array}$ & 15.9 & 0 & - & 41.0 & 42.9 & - & 0.716 \\
\hline
\end{tabular}

CES-D = Calificación total de prueba de depresión. $\mathrm{AD}=$ Afecto deprimido. $\mathrm{SS}=$ Síntomas somáticos. $\mathrm{AP}=\mathrm{Afecto} \mathrm{positivo}$. $\mathrm{RI}=$ Relaciones interpersonales. Número de ajustes = Ajustes aceptables y excelentes referenciados según la enumeración en la Tabla 4 con negrita. Depresión = Dimensión de depresión utilizada según el número de ajuste. Discapacidad= dificultad en caminar 400m. AUC-ROC = Área bajo la curva ROC.

\section{DISCUSIÓN}

En nuestro conocimiento este es el primer estudio con árboles de decisión y regresiones lineales para predecir la restricción en el espacio de vida. A partir del análisis de árboles de decisión (no lineal), es evidente que el EV tiene predictores diferenciales según el sitio y el sexo. En los dos sitios la discapacidad de movilidad y la edad son predictores importante de restricción del EV, tanto en hombres como mujeres. En Manizales, los ajustes 4, 5, 8 y 12, que involucran a las mujeres, asignaron la mayor importancia en las variables depresivas y funcionales, mientras que en los hombres esta importancia estuvo distribuida entre la edad y las dimensiones de depresión. En Natal, desde la perspectiva no lineal, 
se evidenció mayor relación en el afecto positivo, depresión y discapacidad con respecto al EV. Las regresiones lineales indican que en ambas ciudades la capacidad funcional representa una alta importancia con respecto a la predicción de EV en ambos sexos, siendo mucho más fuerte en Natal. También es evidente que hay más restricción del espacio de vida y mayor depresión en mujeres en ambos sitios.

La relación entre EV y discapacidad de movilidad, tal como muestran los resultados de este estudio en los dos sitios, ha sido ampliamente documentada y es claro que las dificultades para caminar o subir escaleras restringen el EV. En la población general de ancianos que viven en la comunidad, ser independiente de las actividades básicas de la vida diaria, incluyendo caminar, se asocia con puntuaciones más altas en el espacio de vida.

Los resultados de diversos estudios sugieren que las restricciones y disminuciones en la movilidad en el espacio de vida pueden ser signos tempranos de una mayor vulnerabilidad a la discapacidad en la vejez (C. L. Curcio et al., 2013; Giannouli et al., 2019; Portegijs et al., 2014, 2016; Rantakokko et al., 2017; Sawyer \& Allman, 2010). Las personas con más altos niveles de funcionalidad pueden tener una mayor movilidad en el espacio de vida simplemente porque tienen una mayor capacidad para moverse. A su vez, el mantenimiento de altos niveles de EV de 60 o más puntos puede ayudar a garantizar mejor función física (Sawyer \& Allman, 2010). En un estudio llevado a cabo en Manizales y Natal se encontró que el EV se relaciona con medidas objetivas y subjetivas de movilidad y discapacidad (C. L. Curcio et al., 2013). Además, las personas con menos de 8 puntos en el SPPB tienen en promedio 10 puntos menos en espacio de vida, y por cada punto adicional de dificultad para la movilidad hay una reducción de 3 puntos en el espacio de vida (C. L. Curcio et al., 2013). Estudios previos han demostrado que, dadas las condiciones medioambientales, los adultos mayores en países en desarrollo requieren altas reservas funcionales para superar las barreras físicas existentes y evitar que su EV se reduzca a medida que envejecen (C.-L. Curcio et al., 2009).

La mayor restricción de EV en la mujeres también está acorde con lo reportado en la literatura (Sawyer \& Allman, 2010). Los factores relacionados con el género (roles de género y desventaja socioeconómica) más que el sexo biológico pueden explicar tales diferencias (Ahmed et al., 2016; Vafaei et al., 2016; M.V. Zunzunegui et al., 2015). Los roles de género en las personas mayores de América Latina implican un entorno hogareño más restringido para las mujeres y fuera del hogar para hombres, ya que las mujeres participan en el cuidado y las tareas domésticas y los hombres son proveedores (Maria Victoria Zunzunegui et al., 2009).

Numerosos estudios han mostrado que la depresión es un predictor independiente de restricción del EV y se ha sugerido que la depresión media parcialmente la relación entre las enfermedades crónicas, las comorbilidades y la movilidad del EV (Baker et al., 2003; Béland et al., 2018; Boyle et al., 2010; C. 
L. Curcio et al., 2013; Peel et al., 2005; Polku et al., 2015; Snih et al., 2012). Los síntomas depresivos pueden ser una expresión de la motivación de la persona mayor para realizar actividades fuera del hogar y conducir a la restricción del EV (Chung et al., 2020). Al igual que en este estudio, una investigación con personas mayores en México reporta que en todos los casos, los efectos de las variables predictivas redujeron el efecto de los síntomas de depresión, y uno de los modelos incluso perdió significancia (González et al., 2013). Esto indica que hay otros factores que afectan negativamente el EV, tal como sucede con la discapacidad de movilidad. Esto no quiere decir que el efecto de la depresión desaparece, solo se reduce, como se pudo observar en los análisis no lineales en los cuales la depresión y algunas de sus dimensiones predicen la restricción del EV. Especialmente en Natal se observa un comportamiento diferencial por sexo, dado que en las mujeres es más importantes el puntaje total de la escala y el afecto positivo, mientras que en los hombres solo fue el afecto positivo. También se observa desde el análisis lineal que en las mujeres resultó significativo los modelos que usaron la calificación total y el afecto deprimido a comparación de los hombres. Además, algunos estudios han encontrado asociaciones de EV y depresión en estudios transversales, pero en análisis longitudinales esta asociación desaparece (Sawyer \& Allman, 2010).

Otro estudio que analiza la movilidad en el EV y las diferentes dimensiones de la depresión muestra, al igual que en este estudio, que la movilidad del EV se asocia con el puntaje total de la escala y todas las dimensiones de la depresión (17). La asociación que mantuvo su significancia por sexo fue la presentada entre la movilidad del EV y los síntomas somáticos y SPPB. Esta asociación es más fuerte en las mujeres, mientras que en los hombres la asociación se relacionó principalmente con el SPPB. En nuestro estudio hay algunas diferencias según el análisis no lineal. Las asociaciones más fuertes con EV en mujeres de Natal fueron la calificación total de depresión y el afecto positivo (modelo 2). En hombres solamente el afecto positivo (modelo 2). En Manizales las relaciones más fuertes tanto en mujeres como en hombres son afecto deprimido, SPPB (modelo 2 y 3 ) y en mujeres síntomas somáticos (modelo 2).

De otro lado, el EV refleja la integración y la participación social en áreas geográficamente definidas, proporcionando una medida de cuán lejos, con qué frecuencia y con qué independencia se mueven los individuos en sus entornos. Los hallazgos diferenciales por sitio pueden incluir múltiples factores además para determinar el EV individual, incluidas las características ambientales y los recursos socioeconómicos. Una mayor restricción del espacio de vida en Natal, especialmente en mujeres, podría explicarse por la mayor proporción de mujeres con depresión, discapacidad de movilidad y mayor número de enfermedades crónicas en ellas. Además, según otros estudios, los participantes de Manizales y Natal carecen de los medios económicos para contrarrestar el efecto de los desafíos ambientales en su EV, por lo que deben depender principalmente de sus reservas funcionales que tal como muestran nuestros 
resultados no son óptimas, y de otro lado, la seguridad personal es un factor importante que puede restringir la movilidad en el EV debido a la alta tasa de criminalidad en estos dos sitios (Auais et al., 2017).

Estudios anteriores han demostrado que las condiciones de salud y las limitaciones en la funcionalidad tienen asociaciones más fuertes con los síntomas somáticos que otras dimensiones de la depresión (Ahmed et al., 2016; Vafaei et al., 2016; Ylli et al., 2016). En nuestro estudio, la relación entre la movilidad en el EV y el afecto deprimido, los síntomas somáticos y el afecto positivo se relacionaron con las dificultades para caminar.

Este estudio tiene algunas limitaciones, si bien los predictores identificados pueden proporcionar pistas de intervención para la recuperación del espacio de vida, no pueden probar causa y efecto debido a su naturaleza transversal. Las personas con mayores niveles de funcionalidad pueden tener una mayor movilidad en el EV simplemente porque tienen una mayor capacidad para moverse, o el mantenimiento de niveles de espacio vital de 60 puntos o más puede ayudar a garantizar la función física. El estudio tiene varias fortalezas, en primer lugar, es un estudio multicéntrico, anidado en un estudio longitudinal. En nuestro conocimiento es el primer estudio que utiliza análisis lineal (regresiones lineales) y no lineal (árboles de decisión), para predecir la restricción en el EV, lo cual permitió tener una visión más completa y detallada del espacio de vida y las variables que lo predicen. El EV mide la movilidad que refleja la "participación" en la sociedad, en lugar de simplemente la capacidad de realizar una actividad específica relacionada con la movilidad, como caminar o subir escaleras. Además, es evidente que el EV tiene predictores diferenciales según el sitio, lo cual sugiere que se deben hacer estudios acerca del papel de las condiciones culturales y geográficas en el EV.

\section{CONCLUSIONES E IMPLICACIONES}

En los dos sitios la depresión, la edad, la discapacidad de movilidad y la funcionalidad son importantes predictores de espacio de vida, principalmente en mujeres. Este conocimiento puede proporcionar información para el desarrollo adicional de intervenciones destinadas a prevenir restricciones en el espacio de vida relacionadas con factores modificables. Nuestros resultados refuerzan la necesidad de implementar medidas para disminuir la depresión y mantener la movilidad y la funcionalidad.

Comprender la asociación entre depresión y EV es vital para la práctica clínica. Las personas mayores con síntomas depresivos pueden ser más difíciles de identificar ya que tienen menos interacciones fuera del hogar, además, la restricción del EV disminuye la autonomía al limitar las oportunidades de ir a dónde, cuándo y cómo se quiera y si se suma la presencia de discapacidad, la 
restricción se incrementa y lleva al círculo vicioso de depresión, discapacidad y restricción de EV, especialmente en mujeres. Es prioritario que el sector público tome acciones en torno a la depresión, funcionalidad y la discapacidad, lo cual se puede materializar a través del fortalecimiento de políticas públicas que abordan estos temas y que permita fortalecer las estrategias preventivas y de detección temprana de ambas condiciones y así disminuir sus consecuencias a largo plazo.

La movilidad en el EV podría ser un objetivo de intervención, ayudando así a disminuir la discapacidad. Las políticas económicas, sociales y de transporte deben considerar el entorno del EV, lo cual tiene implicaciones para la movilidad diaria de las personas mayores y, en consecuencia, para la discapacidad. Mantener la movilidad del EV puede ser valioso para las políticas de salud pública destinadas a fomentar el envejecimiento saludable. Además, el cuestionario de evaluación del espacio de vida es útil en la práctica clínica para estimular hábitos y actividades de caminata en áreas abiertas y seguras, y su monitoreo puede identificar a las personas mayores en riesgo de discapacidad y depresión. 


\section{REFERENCIAS}

Ahmed, T., Vafaei, A., Auais, M., Guralnik, J., Zunzunegui, M. V., T., A., A., V., M., A., J., G., Ahmed, T., Vafaei, A., Auais, M., Guralnik, J., \& Zunzunegui, M. V. (2016). Gender roles and physical function in older adults: Cross-sectional analysis of the International Mobility in Aging Study (IMIAS). PLoS ONE, 11(6), no pagination. https://doi.org/http://dx.doi.org/10.1371/journal.pone.0156828

Allman, R. M., Sawyer, P., \& Roseman, J. M. (2006). The UAB study of aging: Background and insights into life-space mobility among older Americans in rural and urban settings. Aging Health, 2(3), 417-429. https://doi.org/10.2217/1745509X.2.3.417

Auais, M., Alvarado, B., Guerra, R., Curcio, C., Freeman, E. E., Ylli, A., Guralnik, J., \& Deshpande, N. (2017). Fear of falling and its association with life-space mobility of older adults: A cross-sectional analysis using data from five international sites. Age and Ageing, 46(3), 459-465. https://doi.org/10.1093/ageing/afw239

Baker, P. S., Bodner, E. V., \& Allman, R. M. (2003). Measuring Life-Space Mobility in CommunityDwelling Older Adults. Journal of the American Geriatrics Society, 51(11), 1610-1614. https://doi.org/10.1046/j.1532-5415.2003.51512.x

Barnes, L. L., Wilson, R. S., Bienias, J. L., Mendes De Leon, C. F., Kim, H. J. N., Buchman, A. S., \& Bennett, D. A. (2007). Correlates of life space in a volunteer cohort of older adults. Experimental Aging Research, 33(1), 77-93. https://doi.org/10.1080/03610730601006420

Béland, F., Julien, D., Bier, N., Desrosiers, J., Kergoat, M. J., \& Demers, L. (2018). Association between cognitive function and life-space mobility in older adults: Results from the FRéLE longitudinal study. BMC Geriatrics, 18(1). https://doi.org/10.1186/s12877-018-0908-y

Bentley, J. P., Brown, C. J., McGwin, G., Sawyer, P., Allman, R. M., \& Roth, D. L. (2013). Functional status, life-space mobility, and quality of life: a longitudinal mediation analysis. Quality of Life Research, 22(7), 1621-1632. https://doi.org/10.1007/s11136-012-0315-3

Boyle, P. A., Buchman, A. S., Barnes, L. L., James, B. D., Bennett, D. A., \& Boyle PA, Buchman AS, Barnes L, James B, B. D. (2010). Association between life space and risk of mortality in advanced age. Journal of American Geriatrics Society, 58(10), 1925-1930. https://doi.org/10.1111/j.15325415.2010.03058.x.

Campo-Arias, A., Díaz-Martínez, L. A., Rueda-Jaimes, G. E., Cadena-Afanador, L. del P., \& Hernández, N. L. (2007). Psychometric Properties of the CES-D Scale Among Colombian Adults from the General Population. Rev. Colomb. Psiquiatr, 36(4), 664-674.

Cardol, M., De Haan, R. J., Van Den Bos, G. A. M., De Jong, B. A., \& De Groot, I. J. M. (1999). The development of a handicap assessment questionnaire: The Impact on Participation and Autonomy (IPA). Clinical Rehabilitation, 13(5), 411-419. https://doi.org/10.1191/026921599668601325

Chung, M. H., Leung, S. F., \& Välimäki, M. (2020). Use of tracking technology to examine life-space mobility among people with depression: A systematic review protocol. In BMJ Open (Vol. 10, Issue 1). https://doi.org/10.1136/bmjopen-2019-034208

Cohen-Mansfield, J., Shmotkin, D., \& Hazan, H. (2010). The effect of homebound status on older persons. 
Journal of the American Geriatrics Society, 58(12), 2358-2362. https://doi.org/10.1111/j.15325415.2010.03172.x

Curcio, C.-L., Gomez, F., \& Reyes-Ortiz, C. A. (2009). Activity Restriction Related to Fear of Falling Among Older People in the Colombian Andes Mountains: Are Functional or Psychosocial Risk Factors More Important? Journal of Aging and Health, 21(3), 460-479. https://doi.org/10.1177/0898264308329024

Curcio, C. L., Alvarado, B. E., Gomez, F., Guerra, R., Guralnik, J., \& Zunzunegui, M. V. (2013). LifeSpace Assessment scale to assess mobility: Validation in Latin American older women and men. Aging Clinical and Experimental Research, 25(5), 553-560. https://doi.org/10.1007/s40520-013-0121-y

Eugenia Alvarado, B., Victoria Zunzunegui, M., Beland, F., Sicotte, M., \& Tellechea, L. (2007). Social and Gender Inequalities in Depressive Symptoms Among Urban Older Adults of Latin America and the Caribbean. The Journals of Gerontology Series B: Psychological Sciences and Social Sciences, 62(4), S226-S236. https://doi.org/10.1093/geronb/62.4.S226

Giannouli, E., Fillekes, M. P., Mellone, S., Weibel, R., Bock, O., \& Zijlstra, W. (2019). Predictors of reallife mobility in community-dwelling older adults: An exploration based on a comprehensive framework for analyzing mobility. European Review of Aging and Physical Activity, 16(1). https://doi.org/10.1186/s11556-019-0225-2

Gomez, F., Zunzunegui, M. V., Alvarado, B., Curcio, C. L., Pirkle, C. M., Guerra, R., Ylli, A., \& Guralnik, J. (2018). Cohort Profile : The International Mobility In Aging Study ( IMIAS ). International Journal of Epidemiology, May, 1-9. https://doi.org/10.1093/ije/dyy074

González, B. C. S., Delgado, L. H., Quevedo, J. E. C., \& Cabriales, E. C. G. (2013). Life-space mobility, perceived health, and depression symptoms in a sample of Mexican older adults. Hispanic Health Care International, 11(1), 14-20. https://doi.org/10.1891/1540-4153.11.1.14

Guralnik, J. M., Simonsick, E. M., Ferrucci, L., Glynn, R. J., Berkman, L. F., Blazer, D. G., Scherr, P. A., \& Wallace, R. B. (1994). A short physical performance battery assessing lower extremity function: Association with self-reported disability and prediction of mortality and nursing home admission. Journals of Gerontology. https://doi.org/10.1093/geronj/49.2.M85

Huang, J., \& Ling, C. X. (2005). Using AUC and Accuracy in Evaluating Learning Algorithms. IEEE TRANSACTIONS ON KNOWLEDGE AND DATA ENGINEERING, 17(3), 299-310.

James, B. D., Boyle, P. A., Buchman, A. S., Barnes, L. L., Bennett, D. A., James, Boyle, B. D., Buchman, P. A., Barnes, A. S., Bennett, L. L., \& David. (2011). Life Space and Risk of Alzheimer Disease, Mild Cognitive Impairment, and Cognitive Decline in Old Age. American Journal of Geriatric Psychiatry, 19(11), 961-969. https://doi.org/10.1097/JGP.0b013e318211c219

Kennedy, R. E., Almutairi, M., Williams, C. P., Sawyer, P., Allman, R. M., \& Brown, C. J. (2019). Determination of the Minimal Important Change in the Life-Space Assessment. Journal of the American Geriatrics Society, 67(3), 565-569. https://doi.org/10.1111/jgs.15707

Kono, A., Kai, I., Sakato, C., \& Rubenstein, L. Z. (2004). Frequency of Going Outdoors: A Predictor of Functional and Psychosocial Change Among Ambulatory Frail Elders Living at Home. The Journals of Gerontology Series A: Biological Sciences and Medical Sciences, 59(3), M275-M280. 
https://doi.org/10.1093/gerona/59.3.m275

Kuspinar, A., Verschoor, C. P., Beauchamp, M. K., Dushoff, J., Ma, J., Amster, E., Bassim, C., Dal BelloHaas, V., Gregory, M. A., Harris, J. E., Letts, L., Neil-Sztramko, S. E., Richardson, J., Valaitis, R., \& Vrkljan, B. (2020). Modifiable factors related to life-space mobility in community-dwelling older adults: Results from the Canadian Longitudinal Study on Aging. BMC Geriatrics, 20(1). https://doi.org/10.1186/s12877-020-1431-5

Mandrekar, J. N. (2010). Receiver Operating Characteristic Curve in Diagnostic Test Assessment. Journal of Thoracic Oncology, 5(9), 1315-1316. https://doi.org/10.1097/JTO.0b013e3181ec173d

Nagi, S. Z. (1976). An Epidemiology of Disability among Adults in the United States. The Milbank Memorial Fund Quarterly. Health and Society, 54(4), 439. https://doi.org/10.2307/3349677

Nolen-Hoeksema, S. (2001). Gender Differences in Depression. Current Directions in Psychological Science , 10, 173-176. https://doi.org/10.1111/1467-8721.00142

Organización Mundial de la Salud (OMS). (2017). La salud mental y los adultos mayores.

Pedregosa, F., Varoquaux, G., Gramfort, A., Michel, V., Thirion, B., Grisel, O., Blondel, M., Prettenhofer, P., Weiss, R., Dubourg, V., Vanderplas, J., Passos, A., Cournapeau, D., Brucher, M., Perrot, M., \& Duchesnay, E. (2011). Scikit-learn : Machine Learning in Python. Journal of Machine Learning Research, $12,2825-2830$.

Peel, C., Baker, P. S., Roth, D. L., Brown, C. J., Bodner, E. V, Allman, R. M., Sawyer Baker, P., Roth, D. L., Brown, C. J., Brodner, E. V, \& Allman, R. M. (2005). Assessing mobility in older adults: the UAB Study of Aging Life-Space Assessment. Physical Therapy, 85(10), 1008-1119.

Polku, H., Mikkola, T. M., Portegijs, E., Rantakokko, M., Kokko, K., Kauppinen, M., Rantanen, T., \& Viljanen, A. (2015). Life-space mobility and dimensions of depressive symptoms among communitydwelling older adults. Aging \& Mental Health, 19(9), 781-789. https://doi.org/10.1080/13607863.2014.977768

Portegijs, E., Rantakokko, M., Mikkola, T. M., Viljanen, A., \& Rantanen, T. (2014). Association between physical performance and sense of autonomy in outdoor activities and life-space mobility in communitydwelling older people. Journal of the American Geriatrics Society, 62(4), 615-621. https://doi.org/10.1111/jgs.12763

Portegijs, E., Rantakokko, M., Viljanen, A., Sipilä, S., \& Rantanen, T. (2016). Identification of Older People at Risk of ADL Disability Using the Life-Space Assessment: A Longitudinal Cohort Study. Journal of the American Medical Directors Association, 17(5), 410-414. https://doi.org/10.1016/j.jamda.2015.12.010

Portegijs, E., Tsai, L.-T., Rantanen, T., \& Rantakokko, M. (2015). Moving through Life-Space Areas and Objectively Measured Physical Activity of Older People. PLOS ONE, 10(8), e0135308. https://doi.org/10.1371/journal.pone.0135308

Rantakokko, M., Portegijs, E., Viljanen, A., Iwarsson, S., Kauppinen, M., \& Rantanen, T. (2015). Changes in life-space mobility and quality of life among community-dwelling older people: a 2-year follow-up study. Quality of Life Research, 25(5), 1189-1197. https://doi.org/10.1007/s11136-015-1137-x 
Rantakokko, M., Portegijs, E., Viljanen, A., Iwarsson, S., \& Rantanen, T. (2017). Task Modifications in Walking Postpone Decline in Life-Space Mobility Among Community-Dwelling Older People: A 2-year Follow-up Study. The Journals of Gerontology. Series A, Biological Sciences and Medical Sciences. https://doi.org/10.1093/gerona/glw348

Ruiz-Grosso, P., Loret de Mola, C., Vega-Dienstmaier, J. M., Arevalo, J. M., Chavez, K., Vilela, A., Lazo, M., \& Huapaya, J. (2012). Validation of the Spanish Center for Epidemiological Studies Depression and Zung Self-Rating Depression Scales: A Comparative Validation Study. PLoS ONE, 7(10). https://doi.org/10.1371/journal.pone.0045413

Saajanaho, M., Rantakokko, M., Portegijs, E., Törmäkangas, T., Eronen, J., Tsai, L. T., Jylhä, M., \& Rantanen, T. (2015). Personal goals and changes in life-space mobility among older people. Preventive Medicine. https://doi.org/10.1016/j.ypmed.2015.08.015

Sawyer, P., \& Allman, R. M. (2010). Resilience in mobility in the context of chronic disease and aging: Cross-sectional and prospective findings from the University of Alabama at Birmingham (UAB) study of aging. In New Frontiers in Resilient Aging: Life-Strengths and Well-Being in Late Life (pp. 310-339). https://doi.org/10.1017/CBO9780511763151.014

Sheppard, K. D., Sawyer, P., Ritchie, C. S., Allman, R. M., \& Brown, C. J. (2013). Life-space mobility predicts nursing home admission over 6 years. Journal of Aging and Health, 25(6), 907-920. https://doi.org/10.1177/0898264313497507

Snih, S. Al, Peek, K. M., Sawyer, P., Markides, K. S., Allman, R. M., \& Ottenbacher, K. J. (2012). Lifespace mobility in Mexican Americans aged 75 and older. Journal of the American Geriatrics Society. https://doi.org/10.1111/j.1532-5415.2011.03822.x

Stalvey, B. T., Owsley, C., Sloane, M. E., \& Ball, K. (1999). The Life Space Questionnaire: A Measure of the Extent of Mobility of Older Adults. Journal of Applied Gerontology. https://doi.org/10.1177/073346489901800404

Taylor, J. K., Buchan, I. E., \& van der Veer, S. N. (2018). Assessing life-space mobility for a more holistic view on wellbeing in geriatric research and clinical practice. Aging Clinical and Experimental Research. https://doi.org/10.1007/s40520-018-0999-5

Thomas, J. L., Jones, G. N., Scarinci, I. C., Mehan, D. J., \& Brantley, P. J. (2001). The utility of the CES$\mathrm{D}$ as a depression screening measure among low-income women attending primary care clinics. International Journal of Psychiatry in Medicine, 31(1), 25-40. https://doi.org/10.2190/FUFR-PK9F6U10-JXRK

Vafaei, A., Ahmed, T., Falcão Freire, A. D. N., Zunzunegui, M. V., Guerra, R. O., Freire, A. do N. F., Zunzunegui, M. V., \& Guerra, R. O. (2016). Depression, sex and gender roles in older adult populations: The International Mobility in Aging Study (IMIAS). PLoS ONE, 11(1), e0146867. https://doi.org/10.1371/journal.pone.0146867

Vaughan, L., Corbin, A. L., \& Goveas, J. S. (2015). Depression and frailty in later life: A systematic review. In Clinical Interventions in Aging (Vol. 10, pp. 1947-1958). https://doi.org/10.2147/CIA.S69632

Viljanen, A., Mikkola, T. M., Rantakokko, M., Portegijs, E., \& Rantanen, T. (2016). The Association between Transportation and Life-Space Mobility in Community-Dwelling Older People with or Without 
Walking Difficulties. Journal of Aging and Health, 28(6), 1038-1054. https://doi.org/10.1177/0898264315618919

Villarreal, M. L., Leeuwen, W. J. D. Van, \& Raul, J. (2011). International Journal of Remote Mapping and monitoring riparian vegetation distribution, structure and composition with regression tree models and post-classification change metrics. January 2015, 37-41. https://doi.org/10.1080/01431161.2011.644594

Xue, Q. L., Fried, L. P., Glass, T. A., Laffan, A., \& Chaves, P. H. M. M. (2008). Life-space constriction, development of frailty, and the competing risk of mortality: The women's health and aging study I. American Journal of Epidemiology, 167(2), 240-248. https://doi.org/10.1093/aje/kwm270

Ylli, A., Miszkurka, M., Phillips, S. P., Guralnik, J., Deshpande, N., \& Zunzunegui, M. V. (2016). Clinically relevant depression in old age: An international study with populations from Canada, Latin America and Eastern Europe. Psychiatry Research, 241, 236-241. https://doi.org/10.1016/j.psychres.2016.04.096

Zunzunegui, M.V., Alvarado, B. E., Guerra, R., Gómez, J. F., Ylli, A., Guralnik, J., Freeman E., Karna G., Deshpande N., Garcia A., Kotecha J., Philips S., Curcio C-L., Freire A., Qirjako G., Freeman, E., Karna, G., Deshpande, N., Garcia, A., ... Qirjako, G. (2015). The mobility gap between older men and women: The embodiment of gender. Archives of Gerontology and Geriatrics, 61(2), 140-148. https://doi.org/10.1016/j.archger.2015.06.005

Zunzunegui, Maria Victoria, Alvarado, B. E., Béland, F., \& Vissandjee, B. (2009). Explaining health differences between men and women in later life: A cross-city comparison in Latin America and the Caribbean. Social Science and Medicine, 68(2), 235-242. https://doi.org/10.1016/j.socscimed.2008.10.031

Zunzunegui, M. V., Béland, F., Llácer, A., \& León, V. (1998). Gender differences in depressive symptoms among Spanish elderly. Social Psychiatry and Psychiatric Epidemiology, 33(5), 195-205. https://doi.org/10.1007/s001270050043 